\title{
"FACTORES QUE FAVORECEN DESARROLLO PROFESIONAL DE CONTADORES AUDITORES EGRESADOS/TITULADOS, PERÍODOS 2009-2012, CASO UNIVERSIDAD PÚBLICA"
}

\author{
“FACTORS THAT FAVOR PROFESSIONAL \\ DEVELOPMENT OF GRADUATED/TITLE \\ AUDITOR ACCOUNTANTS, PERIODS 2009-2012, \\ PUBLIC UNIVERSITY CASE"
}

\author{
Marcela Fernández Rozas ${ }^{1}$, Marlene Piña Galdames ${ }^{2}$, Rosemary Ríos Orrego ${ }^{3}$
}

\section{RESUMEN}

Chile en la actualidad se ha enfrentado a diversos cambios en el ámbito laboral, originados por la globalización y el avance de las tecnologías de la información, caso muy reciente es la adopción de las Normas Internacionales de Información Financiera (NIIF/IFRS). Es por esto que hoy en día las funciones de los contadores auditores son cada vez más estandarizadas y exigentes a nivel nacional e internacional, tales cambios exigen que estos profesionales tengan un adecuado desarrollo profesional, para estar acorde con la evolución que han tenido las organizaciones en las últimas décadas.

Si bien la institución en estudio ha realizado algunas investigaciones relacionadas con la empleabilidad de sus egresados, aún falta por ahondar más en relación al desarrollo profesional y si las habilidades que fueron alcanzadas durante su trayectoria académica son suficientes para enfrentar en forma eficaz el campo laboral. El propósito fue realizar un análisis de aquellos factores que favorecen o desfavorecen el desarrollo profesional de los egresados de la organización en estudio, una vez que se insertan en el mundo laboral, específicamente durante sus primeros tres años laborales para las cohortes 2005-2008, titulados período 2009-2012.

El enfoque de este estudio fue de carácter cualitativo, con un alcance de sintetización, se elaboraron entrevistas semiestructuradas en profundidad para egresados/titulados, académicos y empleadores y se revisaron las bases de datos institucionales de la universidad pública.

Los resultados obtenidos indican que los factores que favorecen el desempeño laboral de los profesionales entrevistados, a nivel personal son, la autoestima, el liderazgo, la adaptación al cambio y motivación, y lo que desfavorece son la inseguridad y la falta de carácter. A nivel laboral favorecen el conocimiento, incentivos, ascensos, entre otros, y los que obstaculizan bajos sueldos, presión, inexperiencia, entre otros.

Palabras claves: Competencias Genéricas y Específicas, Empleabilidad, Factores Facilitadores y Obstaculizadores.

Recepción: 9/11/2016. Aprobación: 13/08//2017.

1 Escuela de Auditoría, Facultad de Ciencias Económicas y Administrativas, Universidad de Valparaíso, Valparaíso, Chile, marcela.fernandez@uv.cl 2 Escuela de Auditoría, Facultad de Ciencias Económicas y Administrativas, Universidad de Valparaíso, Valparaíso, Chile, marlene.pina@uv.cl 


\section{ABSTRACT}

Currently, Chile has faced diverse changes in the labor scope, originated by the globalization and the advance of information technologies, a very recent case is the adoption of the International Norms of Financial Information (NIIF / IFRS). Because of it, nowadays auditor accountants functions are more and more standardized and demanding at national and international level, such changes demand that these professionals have a suitable professional development to be agreed with the evolution that the organizations have had today.

Although the institution in study has made some investigations related to its graduated students' employability, it is still necessary to deepen more in relation to the professional development and to make sure if the abilities that were reached during their academic trajectory are enough to face the labor field successfully. The intention was to make an analysis of those factors that favor or work against the professional development of the graduated students of the organization in study, once they are inserted in the labor world, specifically during their first three labor years for cohorts 20052008, titled since period 2009-2012.

The approach of this study was qualitative, with a sintetization reach, semistructured interviews were elaborated in depth for graduated/titled, academic and employers, besides, institutional data bases of the public university were reviewed.

The gotten results indicate that the factors that favor the labor performance of the professionals interviewed, at personal level are, the self-esteem, the leadership, the adaptation to changes and motivation, and what it works against this are the insecurity and the lack of character. At labor level they favor the knowledge, incentives, promotions, and others, and those that prevent this are low pays, pressure, inexperience, and others.

Keywords: Generic and specific competitions, employability, facilitador and obstructive factors.

\section{INTRODUCCIÓN}

El Contador Público y/o Contador Auditor (CPA), según la Comisión Nacional de Acreditación, Chile Acreditación (2007), es un profesional experto en materias de control que, con sólidos conocimientos científico técnicos y una formación integral, está capacitado para participar activamente en el diagnóstico, diseño, evaluación, construcción, mantención y evaluación de sistemas de información y control relacionados con la gestión administrativa de organizaciones de cualquier naturaleza. De esta forma, El CPA elabora e interpreta informes de gestión y es responsable de emitir opinión independiente respecto de los estados financieros de acuerdo a principios y normas profesionales vigentes, asegurando a la sociedad la confiabilidad de ellos.

Las actividades del profesional CPA se encuentran regidas a nivel mundial bajo las Normas Internacionales de Información Financiera, las cuales son establecidas por la Junta de Normas Internacionales de Contabilidad (International Accounting Standards Board, IASB) en el año 2011. A su vez, también se rigen por las Normas Internacionales de Auditoría, que son pro- nunciamientos relacionados con las funciones de auditoría emitidas por el Comité denominado IAASB (International Auditing and Assurance Standards Board o Consejo de Normas Internacionales de Auditoría y Aseguramiento) en el año 2003. Estas normas representan disposiciones reconocidas y aceptadas por la profesión organizada, en torno al Colegio de Contadores de Chile A.G. desde el año 2009.

Existen diversas normas emitidas en relación al desarrollo profesional y el actuar que un Contador Público Auditor tiene, entre ellas las Normas internacionales de Educación y Formación (2004), emitidas por la IAESB (Comité Internacional de Estándares sobre Educación Contable), que establecen un punto de calificación en el que se deberán evaluar las capacidades y competencias mínimas que debe demostrar una persona para ser considerada como un contador profesional, las cuales se abarcarán en la presente investigación, para analizar su importancia en la determinación de los factores relevantes que influyen en el desarrollo profesional de un contador Auditor a nivel nacional y dentro de la universidad en estudio.

El profesional Contador Público Auditor de 
hoy en día se ve obligado a enfrentar a un mundo laboral cada vez más competitivo y exigente, con una normativa internacional más estandarizada a nivel mundial y que está afecta a constantes cambios y actualizaciones, lo que origina que exija cada vez mayores competencias y habilidades que permitirán una rápida inserción laboral y un desarrollo profesional adecuado. Éstas están definidas por diversos factores, tales como las características personales, las habilidades desarrolladas dentro de la Universidad, como también nuevos aprendizajes obtenidos en su área de trabajo. El estudio de tales elementos, pueden servir de herramienta para analizar y determinar cuáles son los aspectos que facilitan u obstaculizan el adecuado desarrollo profesional.

El desarrollo profesional suele tener una serie de significados, ya que es difícil de generalizar o aplicar a todos los tipos de personas. Imbemón (1989) señala:

"Desarrollo profesional es sinónimo tanto de perfeccionamiento, como de formación permanente, reciclaje o entrenamiento, a pesar de que cada una de estas voces responde a significados diferentes, ya que se enmarcan en enfoques teóricos diversos que han ido surgiendo y evolucionando desde la segunda mitad de este siglo). Para otros, desarrollo profesional es un término que sólo cobra sentido en una determinada concepción teórica, que se vincula con un enfoque práctico o crítico del currículum, con una concepción interpretativa de la investigación educativa, con un perfil del docente como agente curricular y con una acepción de la escuela como el centro y la unidad de cambio" (Citado por García, 1999, p. 175).

Las personas, cuando inician una carrera profesional, cuentan con una predisposición determinada, experiencia previa, entre otros factores, los cuales incidirán en sus expectativas y condiciones a futuro. El ámbito laboral puede ayudar a desarrollarse profesionalmente de manera adecuada, pero también pueden influir otros factores claves para el desarrollo profesional, tales como las características propias e inherentes de cada persona como también el crecimiento en el ámbito académico, a través del desarrollo de habilidades técnicas o específicas.

Algunos obstaculizadores a los que se enfrenta una persona a un nivel profesional, según Kelly y Guy (1991), son las obligaciones familiares (en el caso de las mujeres) ya que de ellas se espera que desempeñe dos papeles: uno en la oficina y otro en la casa, siendo que las que tienen obligaciones familiares menores son las que se desempeñan mejor en niveles altos de supervisión, dando por hecho de que deben sacrificar su vida personal para lograr el éxito laboral (citado por Madero, 2009, p. 5).

Otro factor obstaculizador del desarrollo profesional, según Margol (1993) es que se carece de planes de carrera formalmente establecidos para los trabajadores, lo que afecta especialmente a las mujeres, ya que no existe un estándar para ambos sexos, ya que según Jaffe (1985) en el caso de las carreras en las mujeres, no cubren el modelo tradicional que se tiene desde la perspectiva masculina en las empresas (Citado por Madero, 2009, p. 6).

En relación a la formación profesional del CPA dentro de la universidad pública en estudio, si bien la organización, a través de sus tesistas y la Unidad de Gestión Pedagógica-Curricular han desarrollado algunas investigaciones en torno a la empleabilidad, aún no se han iniciado estudios relacionados con el desarrollo profesional de los estudiantes egresados y/o titulados de la carrera de Auditoría.

La hipótesis de trabajo que guío esta investigación fue: "Los aspectos que favorecen u obstaculizan el desarrollo profesional en el mercado laboral de los contadores auditores de una universidad pública dependerán tanto de aspectos personales, académicos como laborales".

En la actualidad se hace indispensable obtener información sobre los resultados en la formación de los egresados de la carrera con el fin de revisar y ajustar el currículo, realizando las mejoras necesarias que permitan el adecuado desarrollo profesional de sus egresados, considerando, además, el cumplimiento de los criterios establecidos en los procesos de acreditación de las carreras.

Por lo anterior es que el objetivo central de esta investigación fue analizar los principales aspectos facilitadores y obstaculizadores que intervienen en el desarrollo profesional del Contador Público Auditor de una universidad pública, período 2009-2012. Además se generaron los siguientes objetivos específicos:

1. Identificar la ruta curricular a los egresados y / o titulados años 2009-2012, durante su estadía en la carrera de Contador Público Auditor en la universidad en estudio, mediante la revisión de las bases de datos universitarias. 
2. Describir desde la perspectiva de los alumnos, académicos y empleadores, el proceso de inserción laboral y desarrollo profesional que han tenido durante los primeros tres años de haber egresado de la carrera, a través de la toma de entrevistas semi-estructuradas las cuales miden aspectos a nivel personal, académico y profesional de los mismos.

3. Distinguir los aspectos facilitadores y obstaculizadores del desarrollo profesional de los alumnos egresados o titulados de los años 2009-2012, a través de entrevistas efectuadas a los alumnos egresados, académicos y empleadores.

4. Explicar los aspectos facilitadores y obstaculizadores del desarrollo profesional de los egresados o titulados de los años 2009-2012, durante los primeros tres años de haber egresado de la carrera.

\section{METODOLOGÍA, MATERIAL Y MÉTODOS}

La presente investigación se realizó bajo un enfoque cualitativo, con un alcance de sintetización, ya que se tiene una visión global del tema, y se debe buscar la información relacionada a las experiencias vividas por los informantes en relación a los factores que desencadenaron una adecuada inserción laboral y desarrollo profesional vividos por ellos una vez que salieron de la Universidad.

La técnica a utilizar en la recopilación y sistematización de información fue la aplicación de entrevistas semi-estructuradas en profundidad a nivel individual y estructurada, realizadas a un grupo de egresados/titulados de la universidad período 2009-2012, pertenecientes a las cohortes 2005 a 2008, teniendo 3 años de egreso como máximo. Además, se realizó una triangulación de las técnicas de recolección de datos, análisis documental y las entrevistas efectuadas a los egresados/titulados de la carrera en estudio.

La metodología se desarrolló en siete etapas: -Recopilación de Información, donde se revisó material bibliográfico relacionado con el desarrollo profesional, a través de la normativa nacional e internacional referente a la formación del Contador Público Auditor, especialmente las Normas internacionales de Educación y Formación para analizar las competencias de este profesional, Visita a Sitios Web que contienen material informativo de datos relacionados con la Universidad en estudio, como también organismos que otorgan información acerca del perfil y competencias del Contador Auditor. -Sistematización de la información recopilada, en la cual se ordenaron los antecedentes mediante narrativas, cuadros y esquemas en general. -Selección del Sujeto de Investigación, este estudio se aplicó a un grupo de egresados / titulados períodos 2009-2012 de la carrera de Auditoría, de las cohortes 2005 a 2008 que están trabajando en diversos sectores económicos. En cuanto a la estructuración del este grupo cubre a una población de 180 alumnos tanto egresados como titulados, y en relación a la muestra a través de la utilización del programa Stats, se determinó a 14 profesionales, con un error muestral del $5 \%$ y grado de confianza del $95 \%$.

Además, se entrevistaron a los empleadores de dicha muestra ya egresados/titulados, como una forma de tener una visión por parte de ellos del desarrollo profesional y analizar además como observan a estos egresados, su desempeño y progreso dentro de la entidad. Por último, se realizaron entrevistas a los informantes claves que son los académicos coordinadores de las líneas de formación del profesional Contador Auditor, de la organización en estudio, para obtener su punto de vista en relación al desarrollo que pueden lograr los profesionales, tanto dentro como fuera de la universidad. -Aplicación de la técnica de recogida de datos y criterios de calidad: La técnica de recogida de datos aplicada es el análisis de información recopilada mediante la realización de entrevistas semi-estructuradas a los sujetos seleccionados, las cuales cumplieron con los criterios de credibilidad (revisión y aprobación por parte de los entrevistados), confirmabilidad (contraposición de las opiniones de los actores que forman parte de la investigación), transferencia (descripción del contexto y caracterización de los sujetos entrevistados) y fiabilidad (validación por parte de juicios de expertos), siguiendo la ruta de las entrevistas respectivas. Análisis y de Discusión de Resultados obtenidos, se contrasta con la recogida de datos mediante las entrevistas y análisis documental realizándose una discusión de información pudiendo analizar la información para compararlos y contrastarlos entre sí. La discusión corresponde al análisis comparativo entre los resultados obtenidos de las opiniones vertidas por los egresados/titulados, académicos y empleadores. Finalmente la última etapa corresponde al -Levantamiento de las Conclusiones, que corresponde a los resultados finales de la investigación, en función de los objetivos propuestos en la investigación. 


\section{RESULTADOS}

Para establecer los conocimientos y habilidades desarrolladas dentro de la formación profesional entregada por la institución en estudio, se hizo una inspección al perfil de egreso y al plan de estudios vigente y también documentación relacionada a su acreditación, entre los períodos 2005- 2008. Con los datos anteriores se logra caracterizar a las cohortes mencionadas, en ellas egresaron 180 alumnos (88\% diurnos y $12 \%$ vespertinos), de los cuales 118 son mujeres y 62 son varones, de ellos se han titulado 111 egresados diurnos, con un mayor porcentaje de varones y 16 titulados vespertinos. La muestra en estudio fue de 14 personas, la que se subdivide en jornadas diurna, que corresponden a 13 personas, y vespertina con sólo 1 . De la jornada diurna, las cohortes de estudio cubiertos fueron el 2005 (con 3 titulados y dos egresado), 2006 (con 5 titulados), 2007 (2 egresados y 1 titulado) y cohorte 2008 (con un titulado). Dentro de esta jornada, hay un total de 6 mujeres y 8 hombres. A su vez, dentro de la jornada vespertina, hay una titulada correspondiente a la cohorte 2005.

El análisis de las competencias y los factores que afectan a estos profesionales, se realizaron mediante entrevistas semiestructuradas a los egresados/titulados, (ver anexo 1), orientadas a investigar cuales fueron sus condiciones de trabajo, las características personales, sociales y ética profesional, como también los factores que facilitaron y/o obstaculizaron su desarrollo profesional dentro de la empresa. De estas entrevistas se desprende que una vez insertos en el ámbito laboral, los egresados/titulados manifestaron haber adquirido nuevas competencias, las que han ido desarrollando especialmente dentro de su propio trabajo. Algunas de las nuevas capacidades que ellos reconocieron son las siguientes:

“E6: La Revisión y aplicación de procedimiento de auditoría en área municipal y cumplimiento de su marco normativo. Revisiones de saldos de cuentas desde un punto de vista macro, como vista inicial antes de la aplicación de los procesos de auditoría. Desarrollo avanzado de Excel".

"E10: En mi caso y en relación al perfil profesional de nuestra carrera he fortalecido $y$ desarrollados conocimientos no tan solo técnicos para el desarrollo de nuestra profesión sino que además la idea y noción de como dirigir y administrar organizaciones...".
De igual manera los egresados también han manifestado un desarrollo en sus habilidades personales. Una opinión importante a destacar:

\begin{abstract}
“E8: No sé si tenga capacidad de liderazgo, puesto que no la he desarrollado en los puestos de trabajo que me he encontrado. Pero algunas competencias las he ido desarrollando en el tiempo y que también pueden ser importantes para ser un buen líder, como saber escuchar y tener capacidad de enfrentarme a un mayor grupo de gente sin problemas".
\end{abstract}

Además se logró entrevistar a seis académicos de la escuela de auditoría en estudio, considerados como informantes claves (Ver Anexo 2), también se entrevistaron a tres empleadores representativos de la muestra en estudio de egresados/titulados, (Ver Anexo 3). Algunas opiniones consideradas significativas desde la mirada de los académicos y de los empleadores:

"A4: Uno de los factores más importantes como fortaleza de los alumnos de la escuela, que tiene que ver con un tema muy personal, que es el esfuerzo y la capacidad de trabajo que traen".

"A6: La participación, porque los chicos si bien es cierto, son buenos profesionales, pero les falta el desenvolverse mejor en el ámbito profesional, que tengan un lenguaje más técnico, obligarlos a la lectura, ...".

"E2: Las mayores potencialidades son la capacidad de aprendizaje, respecto a los conocimientos técnicos no se puede tener una claridad respecto a temas específicos, pero en forma general cumple con los conocimientos requeridos para su cargo, que abarcan en su mayoría temas contables".

"E1: En general se adaptan bien, pero en algunas personas se ve complicado en las habilidades blandas, las cuales puede irse trabajando en esto, mediante la misma experiencia profesional".

"E2: Se dificulta por el nivel poco extrovertido puede afectar el modo de desarrollo en clientes".

A continuación se presentan en las Figura 1, 2 y 3 los aspectos que facilitaron u obstaculizaron el desarrollo profesional desde la perspectiva de cada uno de los informantes, los cuales tomaron en cuenta los aspectos personales, laborales y académicos, desde la mirada de los egresados/ titulados período 2009-2012, académicos y empleadores, para las cohortes del 2005-2008. 
Figura 1: Aspectos que favorecen y obstaculizan el desempeño profesional, desde la perspectiva de los egresados/titulados.

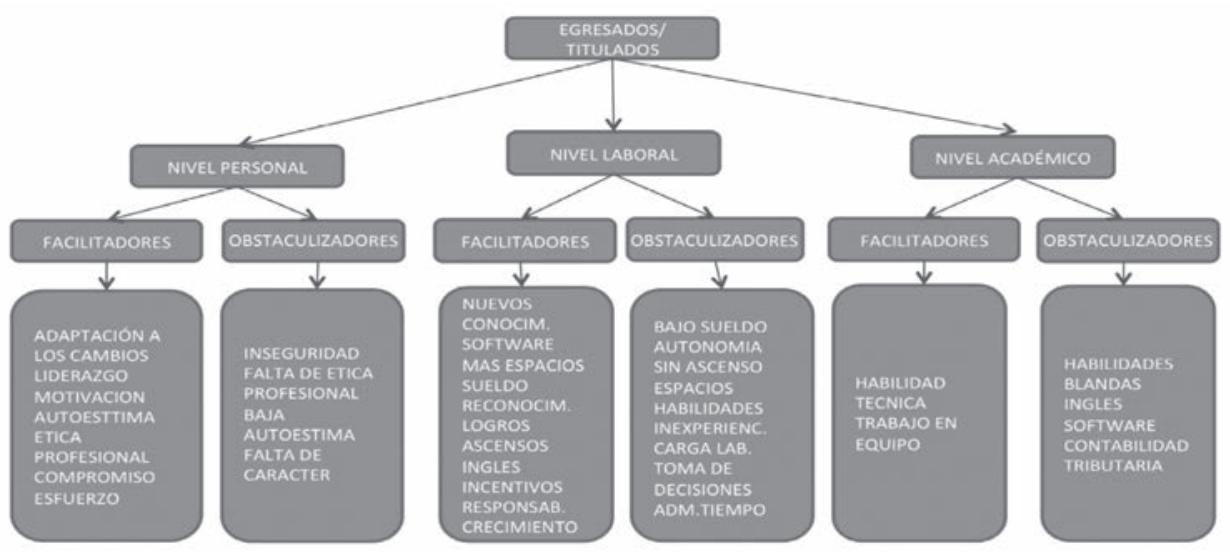

Fuente: Elaboración propia 2016, a partir de las entrevistas realizadas.

Figura 2: Aspectos facilitadores y obstaculizadores del desarrollo profesional desde la perspectiva de los Académicos.

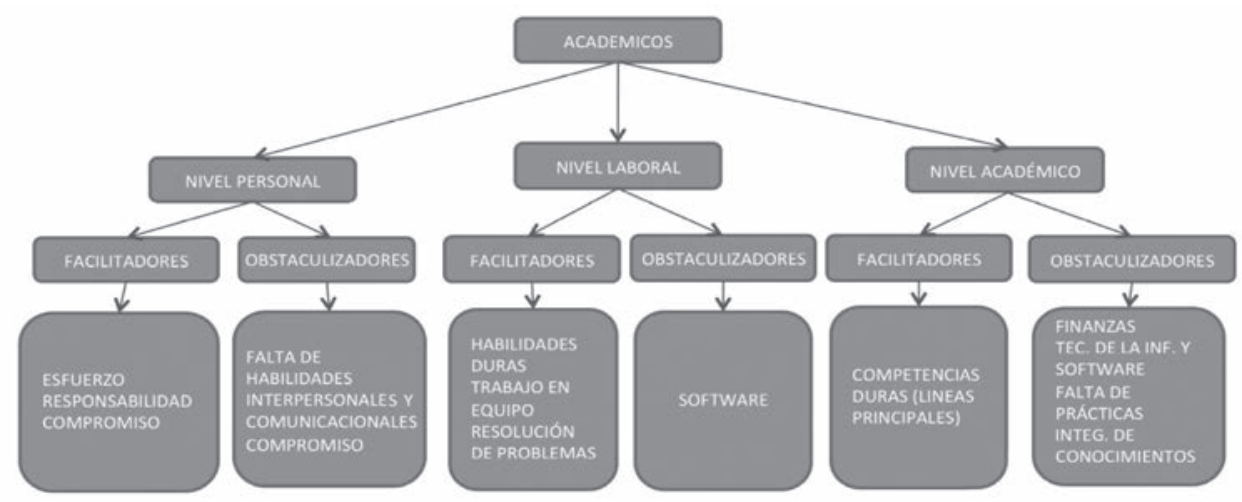

Fuente: Elaboración propia 2016, a partir de las entrevistas realizadas.

Figura 3: Aspectos que favorecen y desfavorecen el desarrollo profesional desde la perspectiva de los Empleadores.

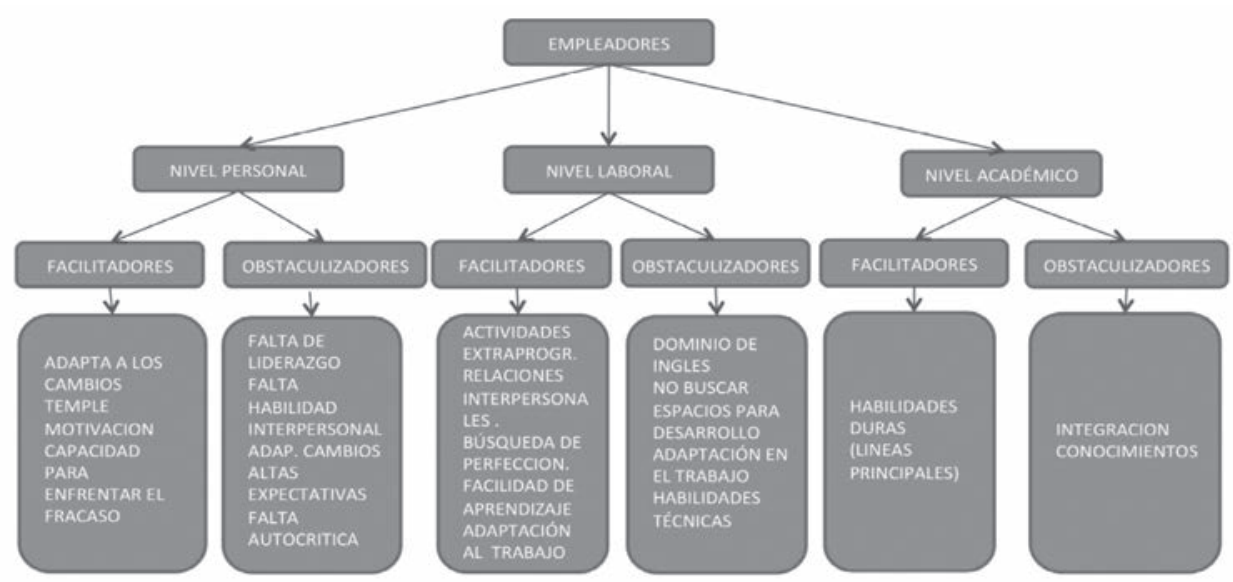

Fuente: Elaboración propia 2016, a partir de las entrevistas realizadas. 


\section{CONCLUSIÓN}

A medida que el estudiante avanza en la malla curricular de la carrera de Contador Público Auditor, adquiere y desarrolla una serie de conocimientos técnicos y habilidades duras, las que le permitirán demostrar un desempeño eficiente en el ámbito laboral, considerándose las más valoradas a criterio según lo señalado por el IASB, de igual manera por la Comisión Nacional de Acreditación y de la Universidad Pública, lo que se establece en el perfil del egresado de la carrera de Auditoría.

Los egresados/titulados concuerdan en competencias que consideran que tienen para desenvolverse en el ámbito laboral tales como las habilidades interpersonales que conlleva a competencias como el liderazgo. Los egresados reconocen contar con esta capacidad, sin embargo los académicos señalan que no es así. Caso contrario es la motivación, que gran parte de los egresados considera que se encuentra conforme con su actual trabajo.

En relación a los obstaculizadores, la inseguridad y la falta de habilidades interpersonales es un factor considerado por gran parte de los académicos y empleadores. Los egresados se sienten un tanto inseguros de sí mismos en el ámbito profesional. A nivel laboral, el trabajo en equipo y el perfeccionamiento (tanto el impartido por la misma organización como el buscado por el egresado) constituyen efectivas formas de potenciar el desarrollo profesional a opinión de los tres tipos de entrevistados. Los empleadores consideran que los egresados aprenden con rapidez dentro de nuevas capacitaciones y softwares, lo que lo consideran un factor a favor. A su vez, los obstaculizadores destacados son la falta de autonomía (considerado por varios de los egresados entrevistados) y también poco incentivo en relación a sus remuneraciones, que no compensan sus funciones desempeñadas dentro de la empresa.

De acuerdo al factor académico, destacan por todos los entrevistados los sólidos conocimientos de las principales ramas de la malla curricular: Tributación, Contabilidad y Auditoría, sin embargo los académicos señalan que estos conocimientos no se integran lo suficiente, tal como se aplica en el ámbito laboral. Junto con esto, se consideran algunas ramas complementarias a la carrera que son insuficientes dentro del plan de estudios, tales como inglés y finanzas. Varios egresados reconocieron ser esforzados, sin embargo los académicos de la Escuela no lo observan de esta forma e indican que deben ser- lo en mayor medida. En relación a la ética, un académico señala que es menester incluirla en la currícula de manera que permita una formación integral. La baja autoestima y la falta de carácter son obstaculizadores reconocidos por algunos de los egresados, que les ha provocado ciertas dificultades en el trabajo.

En relación a las competencias a nivel laboral, dentro de las más valoradas de acuerdo a la opinión de los entrevistados se encuentran la experiencia laboral previa, especialmente en funciones relacionadas a la carrera, lo que les permite un aprendizaje más rápido una vez insertos en el ámbito laboral. Además también ayuda perfeccionarse y tener nuevos conocimientos técnicos, tanto dentro de la empresa como también que el propio profesional busque las instancias para desarrollar nuevas habilidades. Esta característica es valorada por parte de los empleadores, ya que ellos fomentan este tipo de actividades. Junto con ello, también se insta a fortalecer las relaciones interpersonales mediante actividades extra laborales, aunque los egresados y titulados cuentan con esta característica a opinión de los entrevistados. Sobre los obstaculizadores a nivel laboral, según lo señalado por los entrevistados, especialmente por los académicos de la Escuela de Auditoría, se encuentran el manejo de idioma inglés, ya que gran parte de los egresados cuenta con un nivel básico y lo convierte en limitante para optar a mejores opciones laborales u oportunidades de ascenso. Algunos egresados observan que lo anterior está condicionado por la subjetividad de los empleadores, la falta de competencias técnicas y el lento crecimiento de la organización.

Dentro de los factores a nivel académico, las mayores competencias con la que cuentan los egresados y titulados son las habilidades duras, especialmente las líneas de auditoría, contabilidad y tributación, aspectos valorados dentro del ámbito laboral y considerados relevantes por parte de académicos y empleadores, aunque algunos de ellos consideran que podrían actualizarse más los conocimientos, especialmente en el área de tributación e IFRS. Dentro de la universidad, los egresados plantean que existen diversas instancias para desarrollar las habilidades interpersonales y comunicacionales, tales como talleres y exposiciones, no obstante, según empleadores y académicos no es suficiente, ya que la carencia de esta competencia es una de las más relevantes en los alumnos cuando recién se insertan en el ámbito laboral.

En relación a los mayores obstaculizadores a nivel académico, es el dominio del idioma in- 
glés, ya que llegan al mercado laboral con conocimiento nulo o muy básico, en que lo hace transformarse en una limitante para optar a mejores cargos dentro de la empresa. Sin embargo, de acuerdo a lo planteado por los empleadores, en algunas organizaciones se puede optar a perfeccionarse en esta área por medio de cursos financiados total o parcialmente por ellas. También, a opinión de los entrevistados, existe la necesidad de implementar herramientas de software dentro de la universidad, como una forma de aplicación práctica de los conocimientos teóricos adquiridos en las clases. Desde la perspectiva de los empleadores no se considera un mayor obstaculizador, ya que existen inducciones y cursos para aprender estas herramientas.

\section{BIBLIOGRAFÍA Y REFERENCIAS}

Comisión Nacional de Acreditación, Chile acreditación (2007) Criterios de Evaluación para carreras de Contador Público y/o Contador Auditor. Santiago de Chile.

Comisión Nacional de Acreditación de Pregrado CNAP. (2007) Criterios de evaluación para carreras de Contador Público y / o Contador Auditor (PDF) Santiago de Chile. Recuperado Diciembre 10, 2013 de http://www.udp.cl/descargas/facultades_carreras/economia/acreditacion/crit ingecom_contador_publico_auditor.pdf

Consejo de Normas Internacionales de Ética para contadores (International Ethics Standards Board of Accountants). Código de Ética para Profesionales de la Contabilidad (PDF) New York. Recuperado diciembre 06, 2013 de http: / / www. ifac.org/sites / default/files / publications / files / Codigo-de-Etica-Code-of-Ethics-Spanish-Translation.pdf.

Federación Internacional de Contadores, International Federation of Accountants, IFAC (2008). Manual de los pronunciamientos Internacionales de Formación, New York USA.

García S. (1999) El Desarrollo Profesional, análisis de un concepto complejo. Recuperado el 04 de Enero de 2014. Disponible en: http: / / www.mecd.gob.es / dctm / revistade-educacion / articulosre318/ re3180907707. pdf?documentId=0901e72b81270bac
Madero, S. (2009) Factores relevantes del desarrollo profesional y de compensaciones en la carrera laboral del trabajador. Monterrey, México.

\section{ANEXO 1: ENTREVISTA SEMIESTRUCTU- RADA DIRIGIDA EGRESADOS Y TITULA- DOS DE LAS COHORTES 2005 A 2008.}

Los objetivos que se buscan cumplir con la presente entrevista se relacionan con analizar cuáles son los aspectos facilitadores y obstaculizadores que intervienen en el desarrollo profesional del Contador Público Auditor de una Universidad Pública, de las cohortes 2005 a 2008.

Se deja claro que los antecedentes recopilados en la presente tesis serán utilizados sólo para fines educacionales.

Objetivo: Describir desde el punto de vista de los egresados/titulados, su proceso de inserción laboral y su visión del desarrollo profesional que han tenido durante los primeros años de haber egresado de la carrera.

a) Datos generales del entrevistado sobre su área laboral.

1. ¿Cuál es el nombre de la empresa en la que se desempeña?

2. ¿Qué cargo tiene dentro de la empresa y dentro de qué área?

3. ¿Cuánto tiempo lleva desempeñando en la empresa?

4. ¿Qué funciones principales debe desempeñar?

5. ¿Tiene personas a su cargo?

6. ¿Usted trabajó durante su período de formación profesional? ¿En qué?

b) Indagación de características del desarrollo profesional (desarrollo de competencias: básicas-genéricas y específicas).

1. ¿Qué conocimientos nuevos ha desarrollado en relación a las áreas específicas consideradas en el perfil profesional?

2. ¿Qué cursos de perfeccionamiento ha participado dentro de la empresa o en forma individual? ¿quién ha asumido los costos?

3. ¿Qué software domina y en qué nivel?

4. ¿Cuál es su nivel de inglés?

5. ¿Ha buscado nuevos espacios para desarrollar nuevas habilidades para tener un mejor desempeño laboral? ¿Cómo cuáles? 


\section{En relación a la ética y valores.}

1. ¿Se siente motivado dentro de su lugar de trabajo? ¿Por qué?

2. ¿Considera que tiene suficiente autonomía? ¿Cómo lo ve reflejado?

3. ¿Qué situaciones en su trabajo le han puesto en situación complicada de acuerdo a sus valores?

c) Indagación de características del desarrollo personal.

En relación a incentivos otorgados por la empresa:

1. ¿La empresa otorga oportunidades de crecimiento profesional? ¿De qué forma?

2. ¿La empresa retribuye económicamente en forma adecuada según su desempeño?

\section{Sobre características personales:}

1. ¿De qué forma se ha adaptado a los cambios? ¿Qué dificultades ha tenido?

2. ¿Qué habilidades de liderazgo tiene?

3. ¿Qué visión tiene de sí mismo?

4. ¿Qué tan fuerte es su autoestima? ¿Por qué cree que es así?

5. Cuando piensa en mejorar su trabajo, ¿considera otros puntos de vista? ¿De qué forma?

6. ¿Cuáles son sus limitaciones?

7. ¿En qué situaciones observa que necesita fortalecer o desarrollar más habilidades personales?

\section{Sobre relación con colegas de trabajo y su- periores.}

1. ¿Cómo describiría la relación con sus superiores a nivel personal y profesional?

2. ¿Se siente cómodo trabajando en esta empresa? ¿Qué situaciones podrían mejorar?

3. ¿Los superiores reconocen los logros de sus empleados? ¿De qué forma?

d) Indagación de características sobre desarrollo social.

1. ¿La empresa fomenta actividades externas al trabajo que faciliten las relaciones entre los empleados? ¿Cómo cuáles?

2. ¿Participa de una instancia colectiva relacionada con su trabajo? ¿Cuál? e) Factores facilitadores del desarrollo profesional.

1. ¿De qué forma la universidad ayuda a potenciar las habilidades interpersonales o genéricas? Por ejemplo: trabajo en equipo, comunicación o liderazgo.

2. ¿Considera que los contenidos estudiados en la universidad han sido suficientes para responder satisfactoriamente en el trabajo? ¿Por qué?

f) Factores que son obstaculizadores del desarrollo profesional.

1. ¿Cuáles cree que han sido las principales falencias que le han ocasionado problemas de integración dentro de su trabajo?

2. ¿Qué características personales han dificultado su trabajo?

3. ¿Nota que en su trabajo existen carencias de oportunidades de ascenso o de mayores responsabilidades? ¿Por qué?

ANEXO 2: ENTREVISTA DIRIGIDA A ACADÉMICOS DE LA ESCUELA DE AUDITORIA, DE UNA UNIVERSIDAD PÚBLICA

Objetivo: Obtener información relacionada a la visión que tienen los docentes de la Escuela, sobre las distintas competencias y debilidades que se observan en los alumnos en su formación como profesional y al momento de insertarse en el ámbito laboral.

a) Antecedentes generales.

1. ¿Dentro de que línea dentro de la malla curricular se desempeña?

2. ¿Qué cátedras imparte?

3. ¿Cuál es su carga horaria?

b) Factores que inciden en desarrollo profesional de alumnos, según visión de docentes (Dentro de la universidad).

1. ¿Cuáles son las principales características (positivas y negativas) que sobresalen en los alumnos recién egresados?

2. ¿Cuáles son las principales competencias que se potencian en la carrera?

3. Con respecto al plan de estudio, ¿qué cambiaría en él y que dejaría?

4. ¿Cuáles son las falencias que usted considera que no han sido erradicadas aún dentro de la formación en la universidad, que se ven reflejadas en los alumnos al enfrentarse al campo laboral? 
ANEXO 3: ENTREVISTA DIRIGIDA A EMPLEADORES DE LOS ALUMNOS EGRESADOS Y TITULADOS DE LA ESCUELA DE AUDITORIA, DE UNA UNIVERSIDAD PÚBLICA

Objetivo: Obtener la visión de los empleadores sobre las competencias profesionales demostradas y las falencias presentadas por parte de los alumnos de la Escuela dentro de su desempeño en la empresa.

a) Antecedentes generales.

1. ¿Cuál es el nombre de la empresa en la que se desempeña?

2. ¿Qué cargo tiene dentro de la empresa y dentro de qué departamento?

Indagación sobre desarrollo de competencias de los egresados y titulados.

1. ¿El empleado es una persona que busca el perfeccionamiento profesional a tanto dentro como fuera de la empresa? ¿De qué forma?

2. ¿Dentro de la empresa el empleado hace uso del idioma inglés? Si es así, ¿Cuál es su nivel demostrado?

3. ¿Qué tal ha sido la facilidad de aprendizaje del trabajador en relación al manejo de software y sistemas de información utilizados?

4. ¿La empresa ayuda a mantener la motivación entre los empleados? ¿De qué forma?

5. ¿De qué forma el trabajador se ha ido adaptando al trabajo? ¿Cuáles han sido sus dificultades?

6. ¿La persona ha manifestado habilidades de liderazgo? ¿De qué forma?

7. ¿El trabajador aprovecha cabalmente las oportunidades de crecer dentro de la empresa?

8. ¿Qué tal ha sido la adaptación que ha tenido él tras nuevos cambios en su trabajo?

9. ¿Cómo es la relación del empleado con sus colegas?

10. ¿Cuáles son las mayores potencialidades y capacidades a nivel de conocimientos técnicos que tiene el trabajador?

11. ¿Qué características del trabajador (a nivel personal y profesional) han dificultado el desarrollo de su trabajo?

12. ¿De qué forma él enfrenta el fracaso o situaciones difíciles? 